\title{
Sparse Voltage Measurement-Based Fault Location Using Intelligent Electronic Devices
}

\author{
Ke Jia, Bin Yang, Xiongying Dong, Tao Feng, Tianshu Bi, and David Thomas
}

\begin{abstract}
This paper proposes a fault-section location method based on sparse measurements, aimed at asymmetrical faults. A virtual current vector is defined to indicate the faulted section, which is sufficiently sparse except that the fault position corresponding entries are nonzero. To simplify the algorithm, the virtual vector is fixed by amplitudes of voltages and impedances and the feasibility is demonstrated. The Bayesian Compressive Sensing theory is introduced to reduce the number of required intelligent electronic devices (IEDs). In addition, the minimal number of IEDs and their allocation are discussed. The performance of the proposed method is validated in a 69-bus, $12.66 \mathrm{kV}$ distribution system with six distributed generations (DGs) in response to various fault scenarios. The simulation results show that the method is robust for single-phase, double-phase, and double-phase to ground faults with high resistance under noisy condition. Furthermore, the method is applicable for networks with inverter interfaced DGs.
\end{abstract}

Index Terms-Fault-section location; sparse measurement; IEDs; BCS theory; DGs.

\section{INTRODUCTION}

$\mathrm{W}$ ITH the development of both distribution automation (DA) and distribution management system (DMS), intelligent electronic devices (IEDs) begin to gather the operation and fault information of networks in real time [1], which promotes the further development of fault location methodology.

To date, fault location methods can be classified into the following categories: impedance estimation methods [2]-[6], travelling wave methods [8]-[13], and wide area measurementbased methods [14]-[32]. Impedance estimation methods calculate the impedance using the post-fault measurement to estimate the distance between fault and observation point. These are sensitive to unpredictable laterals and load taps of the distribution networks [7]. Travelling wave methods utilize the time of propagation and reflection of high frequency wave to evaluate the position of the fault [28]. Wide area measurementbased methods utilize wide area measurement to locate the fault with assistance of intelligent devices and algorithms. The influence of load change is crippled and injection of high frequency signals is avoided in wide area measurement-based methods; therefore, these have been promoted in recent years.

Wide area measurement-based methods determine the faulted area using the synchronous fault information gathered from several measurements. In [14]-[18], the fault location is obtained by monitoring voltage sags at different nodes. It is necessary to simulate different fault scenarios and then compare the simulation results with the measured voltages to select the optimal match. Reference [19] combines voltage sag methods with impedance estimation methods, effectively decreases "pseudo" impedance estimations, and proposes a systematic method to induce low voltage areas. Currently, fault indicator (FI)-based methods have received a new focus [20]-[22]. The faulted area determined by these methods [14]-[22] relies on the position of the monitoring device. Insufficient investment would definitely increase the range of the determined faulted area, thus increasing troubleshooting time.

IEC 61850, published in 2003, specifies the communication system for power utility automation [23]. Since then, efforts to improve the fault location function of DMS have been made [24]-[26]. However, over investment of IED definitely leads to costly deployment and high bandwidth communication networks.

Several researches improved the location performance using synchronous phasors, measured by phasor measurement units (PMUs) [27]-[31]. However, the upgrading of facilities can only promote the accuracy of methods without solving the problem of the large determined range.

To solve this problem, a series of methods based on an automatic algorithm have been proposed. The introduction of the intelligent algorithm [32]-[35] decreased the operation time, but the algorithm's pre-training and modification to various conditions requires considerable unavailable data. The methods based on iterative state estimation [36]-[39] are universal but cause high computational burden. Reference [40]-[42] mentioned fault location methods based on probability theory, which inspires research on automatic fault location methods.

This study proposes a sparse measurement-based faultsection location method aimed at asymmetrical faults in distribution networks (three-phase faults rarely happen in a distribution system), which is able to narrow the location range down to two adjacent nodes by few IEDs. A defined sparse vector, whose items are a virtual nodal current injection of negative sequence, is proposed as substitute of the true fault current to indicate the faulted section. The virtual current vector is obtained via multiplying the negative sequence node voltage vector with the negative sequence admittance matrix. Given that the purpose of the operation is to obtain an index vector instead of a real current phasor, the operation was simplified by using the amplitudes of voltage and admittance to reconstruct the target vector, and the feasibility analysis is presented. The equations are underdetermined due to the sparsity of measured

This work was supported by the National Key Research and Development Program of China under Grant 2018YFB0904100, by the National Natural Science Foundation of China under Grants 51725702, and 51777071, and by Young Elite Scientists Sponsorship Program by CAST. (corresponding author: Ke Jia.) 
voltages. To restructure the fault injection current vector, Bayesian Compressive Sensing (BCS) theory was introduced. The proposed method is tested in a 69 -bus, $12.66 \mathrm{kV}$ distribution system with six distributed generations (DGs) built in PSCAD/EMTDC according to actual distribution networks. Simulation results show that the proposed method offers good accuracy, adaptation to noise, and large fault resistance. Furthermore, the method is available to distribution networks with inverter interfaced DGs. Via both experiments and analysis, the minimal amount of IEDs was determined by gross nodes quantity and non-zero entries of the index vector. The allocation of IEDs is also discussed.

Compared to existing wide area measurement-based methods, the main contributions of this study are as follows:

- The proposed method is capable to point out the faulted line section from a large scale high- or middle-voltage (above $10 \mathrm{kV}$ ) distribution network, using measurements gathered from very few measuring devices. The requirement of the method for IEDs is far less than that of the existing methods [14]-[22],[24]-[26]. Moreover, allocation of IEDs has been analyzed in this study.

- Negative voltage amplitudes extracted from the original sampling are processed to locate the fault section, instead of the voltage phasors. Furthermore, the post-fault voltage data and network parameters required in the method are obtainable. Therefore, the proposed method avoids the polling of unavailable data [32]-[35] and the investment of PMU [27]-[31].

- Compared to the methods mentioned in references [40][42], an efficient algorithm BCS is adopted to help obtained the position of the fault, which demonstrates improved applicability and anti-noise performance.

- The operation time of the algorithm in this method only scores $\mathrm{ms}$ in RTDS. Compared to computationally burdensome methods [36]-[39], the fault location time is reduced.

- The negative sequence component is used in the proposed method, which makes the fault location result less affected by DG, which normally uses eliminating negative sequence control to maintain a smooth fault ride.

\section{NEW FAULT-SECTION LOCATION METHODOLOGY}

When a permanent asymmetrical shunt fault has occurred in a distribution network, the negative sequence component of the fault current cannot be directly used to indicate the fault position because the post-fault structure is unknown. Therefore, a virtual current vector for indication is proposed in this section.

\section{A. Virtual negative sequence injection current}

Given an N-nodes distribution network, an asymmetric fault occurred on line $l-m$, away from the node $l$ by $c / 1$ length of the line $(0 \leq c \leq 1)$. Assume that the parameter of lines distributes evenly. The actual negative sequence current is as shown in Fig. 1 , presented as a solid arrow.

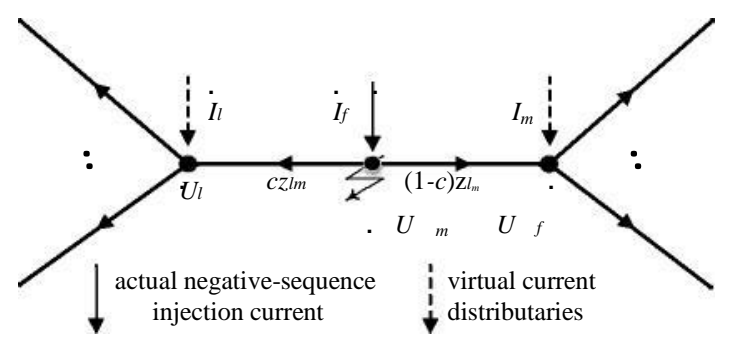

Fig. 1. Negative sequence current equivalent injection.

According to Kirchhoff's Current Law (KCL), the sums of the actual negative sequence current on the nodes $l$ and $m$ are zero. This relationship of currents can be represented via nodal negative sequence voltages as:

$$
\begin{aligned}
& \left.{ }_{l}^{(} U \quad \sum^{n} y_{l}-U\right)+{ }_{i} y(\underset{l}{U} \square-0)+y_{m}\left(U_{l}-\square_{f} U\right) / c=0
\end{aligned}
$$

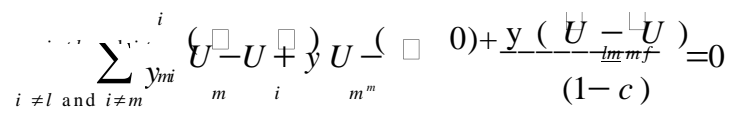

where $y_{l i}\left(y_{m i}\right)$ represents the admittance between node $l$ and each node connected to $l$, represented by letter $i$ ( $m$ and $i), y l m$ represents the admittance of line $l-m, y l\left(y_{m}\right)$ the admittance of the load connected to the ground at node $l(m)$, and $\mathrm{Ut}$ the negative sequence voltage of node $i$ in phasor form. As mentioned above, the exact position of the fault is unknown; therefore, it is difficult to obtain the actual if. Equivalently, it can be substituted by two virtual components $i$ tand $i_{m}$, i.e. the dashed arrows in Fig. 1, separately injected into nodes $l$ and $m$.

The transformation cannot change the rest of the distribution network; therefore, the negative sequence current injections of other nodes remain zero. On this basis, the post-fault topology of the system can be seen as identical to the pre-fault; consequently, virtual negative sequence currents can reasonably be fixed by pre-fault system parameters and post-fault nodal negative sequence voltages:

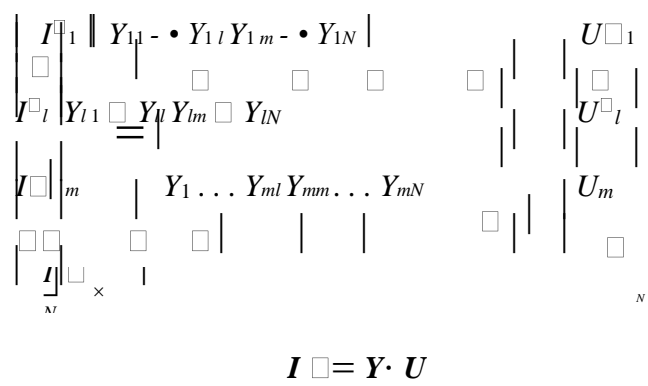

where I represents the virtual nodal negative sequence currents vector in phasor, $U$ represents the nodal negative sequence voltages vector in phasor, and $\boldsymbol{Y}$ represents the negative-sequence admittance matrix of pre-fault networks. ( In reference to [43], the relation of entries in $\boldsymbol{Y}$ with the admittance in (1) is: I n

where $Y_{y k}$ represents the diagonal elements in $\boldsymbol{Y}, y_{k k}$ the diagonal 


$$
\begin{aligned}
& \underset{k k}{Y}=y+\sum y \\
& Y=-y=Y
\end{aligned}
$$




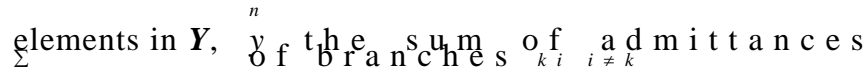
connected to node $k$, and $y_{k}$ the admittance of the load connected to ground at node $k$. With regard to both virtual currents (negative sequence), i.e. the dashed arrows in Fig. 1, these can be seen as two independent current-sources with unknown amplitudes. Based on (2-b) and (3), the virtual current $h$ can be represented as:

$$
\begin{aligned}
& I \square{ }^{n} \\
& { }_{i \bar{l}}=\sum Y U_{l i i} \\
& \left(\begin{array}{c}
y+ \\
y
\end{array}\right)^{+} \sum_{i \neq l} \underset{l i l}{\square}+\left(\sum_{i \neq l}^{n} y \underset{l i i}{U} \square\right) \\
& \sum_{n}-Y\left(U_{U}\right) \boxminus \quad Y \quad U \square U \square
\end{aligned}
$$

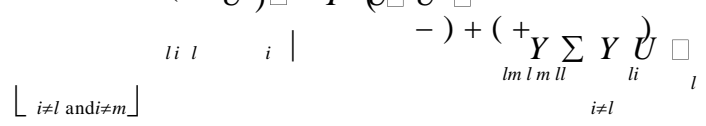

In the same way, $1 m$ can be represented as:

$$
\begin{aligned}
& \text { Integrating (1) with (4) and (5), hand }{ }^{\prime} m \text { can be deduced as: }
\end{aligned}
$$

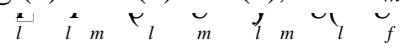

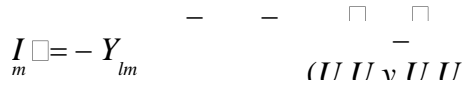

$$
\begin{aligned}
& \begin{array}{c}
I=\sum[(\stackrel{n}{\square} \square)-Y(U U\rceil \\
Y U-U
\end{array} \\
& \text { m }
\end{aligned}
$$

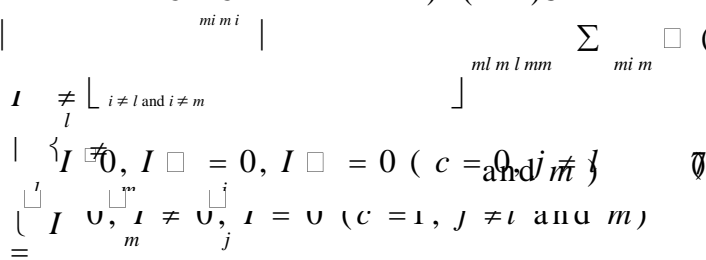

The coefficient $c$ varies with changing fault position. If the value of $c$ ranges between 0 and 1, the fault position is between nodes land $m$; however, if $\mathrm{y}$ is equal to 0 or 1 , the fault occurred on the nodes $l$ or $m$. This can be summarized as:

$0,{ }_{m} I \neq 0, I=0 \quad(0<j<<1, j \neq l$ and $m)$

Note that the vector consisting of $h, \quad i$; is a fictitious current vector, named $1 \mathrm{~F}$.

IEDs cannot be allocated on every bus in the networks; therefore, the measurement $i_{\mathrm{f}}$ is sufficiently sparse. Extract the voltages of measurable buses from $i_{\text {f }}$ to form a new vector $U_{M}$. Accordingly, extract the corresponding rows of the impedance matrix (the inverse of $\boldsymbol{Y}$ ) to form a new matrix $Z_{M}$. Consequently, the virtual vector $I_{F}$ can then be obtained by:

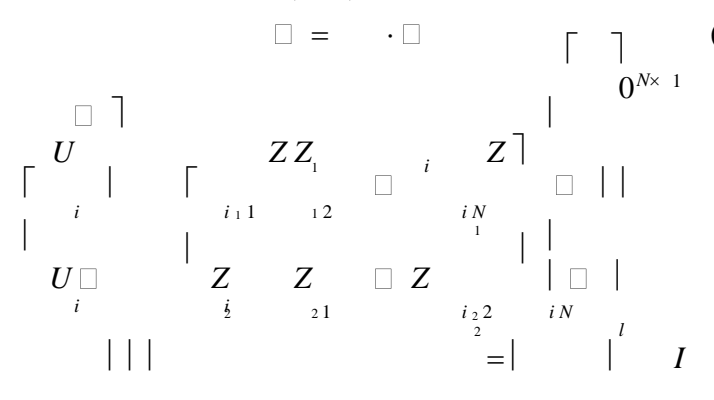

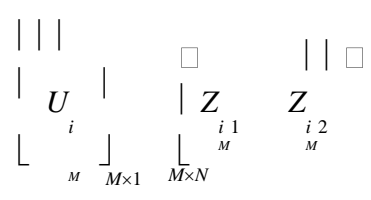

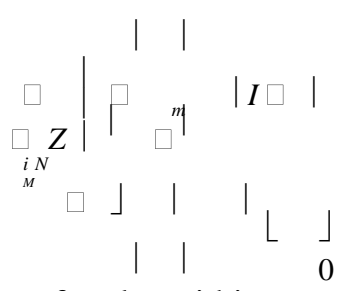

where $N$ represents the gross number of nodes within the network and $M$ represents the number of IEDs.

Only if elements of the vectors are phasors, the equation holds. However, PMU is too expensive to be promoted in 
distribution networks. Therefore, the feasibility of using amplitudes is demonstrated in the next subsection.

\section{B. Negative sequence equation with amplitude elements}

To avoid phasor calculation and rigorous synchronization of the gathered data, this subsection analyzes the feasibility of substituting phasors with their magnitudes based on the property of the bus impedance matrix.

The branches in the network model are classified into two types: 1) tree branches, all distribution lines can be regarded as tree branches due to the radial topology of the distribution networks; 2) link branches, the loads and DGs can be seen as link branches. In the case presented in subsection II-A, assuming that node $l$ is closer to the external grid, the region among them is defined as upstream, and the region behind node $m$ is defined as downstream as shown in Fig. 2.

\section{Fig. 2. Region partition by line 1-m.}

According to the fundamental of the modifying impedance matrix by adding the branch, the modifications of tree and link branches are different.

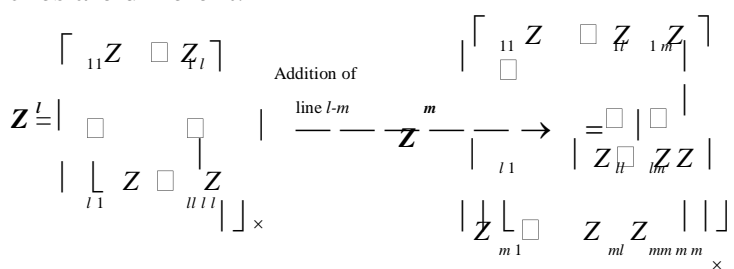

After addition of a tree branch $l-m$ at node $l$, the impedance matrix $\boldsymbol{Z}^{l}$ is modified into $\boldsymbol{Z}^{\boldsymbol{m}}$. The relationships among the elements in $\boldsymbol{Z}^{m}$ are: $Z_{m i}=Z_{l i}, Z_{i m}=Z_{i l}, Z_{m m}=Z l l+{ }_{z l m}$, ${ }^{\text {where }} \mathrm{zlm}$ represents the impedance of line $l-m$ and the letter $i$ represents the upstream nodes.

Similarly, after adding a tree branch downstream, the relationships of the elements in the new matrix are: $Z_{j l}=Z_{m l}$, $Z_{j m}=Z_{m m}, Z_{i j}=Z_{i l}$, where the letter $j$ represents the downstream nodes. The property can be summarized as:

$$
\frac{Z l l}{Z \operatorname{lm}}=\frac{Z i l}{Z i m Z m m} \quad Z^{j m} Z^{{ }^{l j}} Z \quad{ }^{i j}
$$

where $i l$, the letter represents upstream nodes, $j m$, the letter represents downstream nodes.

While adding a link branch does not change the dimension of $\boldsymbol{Z}$, it can only lead to changes on the values of the entries relevant for downstream [44]. For example, with regard to a load connected at node $x$, this link branch can be considered as a branch link between node $x$ and ground defined as the node zero. Therefore, the modification of relevant downstream entries $Z_{i j}$ is:

$(Z-Z)(Z-Z)$

$\underset{x x}{Z}+\underset{0}{Z}-2 \underset{x}{Z} Z_{0}+\underset{x}{Z}$

$$
\begin{array}{llll}
i x & i 0 \quad x j & 0 j
\end{array}
$$

where $Z_{i i}$ represents the modified element $z_{i j}, i, j$, number 0 represent the node represents ground, and $z \times 0$ represents the 
equivalent impedance of the load. According to the definition of self-impedance and mutual-impedance [43], $Z_{i 0}=Z_{0 j}=Z_{00}=$ $Z_{x 0}=0$. Therefore, (11) can be simplified as:

$$
Z_{i j}=Z_{i j}=Z_{-}^{Z} \frac{Z_{x x j}}{Z_{x}+Z_{n} \quad i j} \quad Z_{i X_{x j}}
$$

where $K=Z x x+z x 0$.

The following investigates whether the first equation of (10) still holds after addition of a link branch. Suppose this proposition holds, which means:

$$
\begin{aligned}
& Z_{l l} \stackrel{\bar{L}}{=}{ }^{i l} \\
& { }_{\text {lm }} \overline{\mathbf{L}}_{\text {im }} \\
& Z_{l l} Z_{\frac{Z_{x}}{} \times Z_{x l}} Z_{i l} Z_{i x} \times Z_{x l}
\end{aligned}
$$

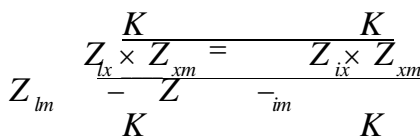

$$
\begin{aligned}
& \text { Z Z Z } \\
& \left(Z_{l l} Z_{i m}-Z_{l m} Z_{i l}\right) K^{2}-\left(Z_{l l} Z_{i x} Z_{x m}+l x x l i m\right. \\
& { }_{l m} i x \times x \text { (14) } \\
& -Z \underset{l x \times m \text { il }}{Z} Z) K+\underset{l x \times l \text { ix xm }}{Z} Z-\underset{l x \times \text { x ix xl }}{Z} Z Z=0
\end{aligned}
$$

where $Z_{l l} Z_{i m}-Z_{l m} Z_{i l}=0$ obviously known from the first equation in (10). Therefore, if the second item of (14) was zero, the proposition would hold. If node $x$ was in upstream:

$$
Z_{i x}\left(Z_{l l} Z_{x m}-Z_{l m} Z_{x l}\right)+Z_{l x}\left(Z_{x l} Z_{i m}-Z_{x m} Z_{i l}\right)=0
$$

If node $x$ was in downstream:

$$
Z_{x m}\left(Z_{l l} Z_{i x}-Z_{l x} Z_{i l}\right)+Z_{x l}\left(Z_{l x} Z_{i m}-Z_{l m} Z_{i x}\right)=0
$$

Integrated with (10), the items in brackets of (15) and (16) are equal to zero. Therefore, this demonstrate the proposition stands, which is the first equation of (10), still holds after addition of a link branch. The other two equations of (10) can also be verified in the same way.

$$
k=\frac{4 l i}{Z_{m}} \bar{Z} \frac{L}{Z_{i} \neq} \frac{m i}{Z_{m m} l} \frac{Z_{j}}{j n}=k_{2}
$$

In conclusion, the relationships among entries of matrix $\boldsymbol{Z}$ can be drawn as:

where $k_{1}$ and $k_{2}$ are dimensionless ratios of impedance.

Therefore, the post-fault node equations implied by (8-b) can be reasonably classified as two types by the different ratios of $Z_{l}$ $Z_{m}$ :

$$
\begin{aligned}
& \mid \quad U Z Z I Z I \\
& \square=\square+\square \quad \mid \int_{i U i}=k_{\mathrm{i}} l_{l}+i_{m}
\end{aligned}
$$

where $U \square_{i}$ represents the voltage of upstream node, $U \square_{j}$ the voltage of downstream node, $I \square_{U i}=U \square_{i} Z_{i m}$ and $I \square_{U j}=U \square_{j} Z_{j m}$. It's a very legible binary linear equation group of $U-I$, in which the two equations are both required for solvability of (18). Namely, for a fault in the network, at least one IED respectively allocated in both up- and down-stream nodes could provide the solvability of (18). One fundamental of the sparse allocation of IEDs for the proposed method is then inferred that the tail ends 
of all feeders should be allocated with IEDs to make sure fault location realized on all lines in network.

Based on (18), a hypothesis is made that there are two scalar quantities $I l^{*}$ and $I_{m}{ }^{*}$ which make the following equations stand:

$$
\Upsilon_{\tau} \mid k_{1} t_{l}+k_{2} I_{l+I_{m}|=| k_{2} \mid I_{l}+I_{m}^{*}}^{+I_{m}} \quad U_{j}
$$

Deduction of (19) seems illogical for vector calculations. However, it makes sense if the two currents are in- or out-phase. They are essentially in-phase which can be inferred from their definition, i.e. two virtual components of actual fault current. Therefore, the hypothesis is founded. Hereto, the mathematical model for fault location has been built as:

$$
\boldsymbol{U}_{M}=\boldsymbol{Z}_{M} \cdot \boldsymbol{I}_{\boldsymbol{F}}{ }^{*}
$$

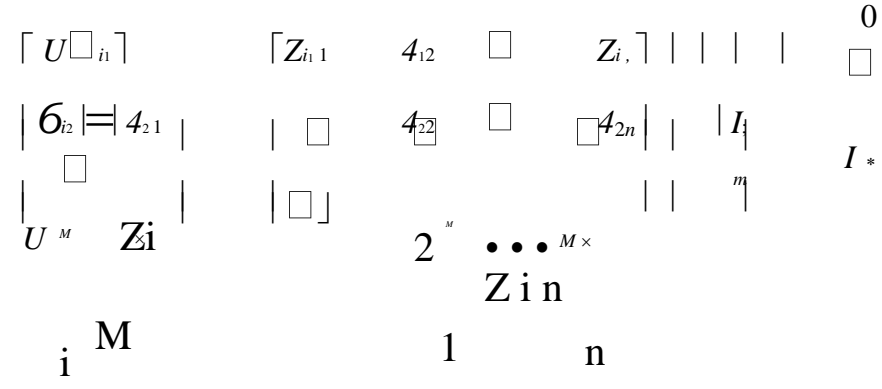

Defining the vector $1 *=\left[\begin{array}{llllll}\mathbf{0} & \ldots & r_{t} & r_{i} \cdot{ }^{\prime} & \ldots & \mathbf{0}\end{array}\right]^{T}$ as the fault position indicator, is able to be fixed by processing RMS of the measured negative sequence voltages and amplitudes of impedances. The two nonzero entries in $1 *$ intuitively indicate the faulted section.

Solving the binary linear equation group (19) wouldn't be straightforward as it looks like. Since the positions of $l$ and $m$ are inconclusive, the $I \square_{U i}$ and $I \square_{U j}$ cannot be certain (e.g. $U \square_{i} Z_{i m}$ the denominator $\mathrm{Zim}$ is related to the position of node

$m$ ). Finding the positions of $l$ and $m$ is an optimization problem, so BCS has been introduced into the proposed method.

\section{Solving process based on BCS Theory}

The Bayesian compressive sensing theory based on Sparse Bayesian Learning (SBL) is more suitable than traditional theories based on $l_{1}$-norm regularization formulation. The reason is that these deal with the perceptual matrix, which has a strong correlation between columns. Furthermore, the SBL algorithm is more inclined to obtain a sparse solution. Moreover, SBL also possesses a stronger anti-noise ability.

The fundamental of compressive sensing theory [45] is:

$$
\mathbf{y}=\boldsymbol{\Phi} \boldsymbol{\theta}+\mathbf{e}
$$

$$
\boldsymbol{\theta}^{\wedge}=\arg \min \{\mathbf{y}-\boldsymbol{\Phi} \boldsymbol{\theta} 2+\rho \boldsymbol{\theta}\}\left(\begin{array}{lll}
2 & 2) \\
1
\end{array}\right.
$$

where $\mathbf{y}$ represents the $M$-dimensional measurement vector, $\boldsymbol{\Phi}$ represents a $M \times N$ matrix defined as sensing matrix $\left(\Phi=\left[\boldsymbol{0}_{1}, \boldsymbol{O}_{2}\right.\right.$, $\left.\left.\ldots, \boldsymbol{0}_{N}\right]\right), \boldsymbol{\theta}$ represents the $N$-dimensional sparse vector to be solved, e represents the $M$-dimensional vector composed of Gaussian white noises, $M<<N$ [46], \|\|$_{p}$ denotes the operation of computing $l_{p}$-norm. The first item in the braces represents the constraint that the deviation between $\mathbf{y}$ and $\boldsymbol{\Phi} \boldsymbol{\theta}$ should be 
minimized, the second item represents the sparsity constraint (i.e. the number of nonzero elements in the vector $\boldsymbol{\theta}$ should be minimized), and $p$ represents a coefficient with which to regulate the relationship of both items, $p>0$.

In this study, the objective function (18) can be described as:

$$
\boldsymbol{U}_{\boldsymbol{M}}=\boldsymbol{Z}_{\boldsymbol{M}} \boldsymbol{I}^{*}{ }_{\boldsymbol{F}}+\mathbf{e}
$$

where e represents the $M$-dimensional vector composed of Gaussian white noises of voltages measurements.

For the initialization of the SBL algorithm, every entry $O_{i}$ in $\boldsymbol{\theta}$ is assumed to obey a Gauss distribution, thus y's Gauss likelihood (GL) model with unknown variance $6^{2}$ can be obtained:

$$
\left.\left(\mathbf{y} \mid \boldsymbol{\theta}, \sigma^{2}\right)\left(2 \pi \sigma_{2}\right)-K^{2} \mathrm{ex}-\frac{\mathrm{p}\left(-2^{1}{ }_{2}\right.}{\mathbf{y}}-\boldsymbol{\Phi} \boldsymbol{\theta}_{\sigma}^{2}\right)
$$

where $6^{2}$ represents the variance of y's GL model.

Equation (24) is a multiple linear regression in which $\mathbf{y}$ is given to estimate $\boldsymbol{\theta}$ and $\sigma^{2}$.

Unlike Laplace Sparseness Prior (LSP), the hierarchical prior based on Relevance Vector Machine (RVM) used in SBL is conjugated to the GL model. The conjugation property greatly reduces the complexity of the algorithm. Hierarchical prior based on RVM is introduced in the following.

Assuming that every element in sparse vector $\boldsymbol{\theta}$ is independent, obeying Gauss distribution with zero mean:

$$
\begin{aligned}
& { }_{i}^{N} \\
& p(\boldsymbol{\theta} \mid \boldsymbol{\alpha})=\prod N(\theta \mid \\
& \left.0, \alpha_{i}{ }^{1}\right) \\
& =1
\end{aligned}
$$

where $\alpha_{i}$ is defined as the precision of

$i=$ Gaussian

density function, i.e. the

reciprocal of variance. Hyper-parameter $\boldsymbol{\alpha}$ is assumed to obey a N

$$
{ }^{\infty} p(\boldsymbol{\theta} \mid a, b)=\prod_{0}
$$

gamma prior distribution:

$$
p(\boldsymbol{\alpha} \mid a, b)=\prod^{N} \text { г }(\underset{i}{\alpha} \mid a, b)
$$

where $a$ and $b$ represent the hyper-parameters of the parameter model of $\boldsymbol{\alpha}$. Marginalizing $\boldsymbol{\alpha}$ obtains the prior distribution of $\boldsymbol{\theta}$ :

Considering $O_{i}$ as measurement data and $N\left(O_{i} \mid 0, \alpha_{i}{ }^{-1}\right)$ as the likelihood function of $O_{i}, \Gamma\left(\alpha_{i} \mid a, b\right)$ is conjugate with $\alpha_{i}$ in the Gauss distribution. Equation (27) satisfies a student- $t$ distribution. The student- $t$ distribution can be very sharp at $O_{i}=0$ if the values of $a$ and $b$ are given reasonably. Therefore, the prior distribution given by (27) ensures that most entries of $\boldsymbol{\theta}$ are 0 ; namely, the sparsity of the reconstructed signal is ensured. Assuming that the reciprocal of the noise variance $f t=6^{-2}$, obeys the Gamma prior distribution, i.e. $p(\boldsymbol{\beta} \mid c, d)=\Gamma(\boldsymbol{\beta} \mid a, b)$, where $c$ and $d$ are hyper-parameters.

Integrated with the discussion of the measurement data $\mathbf{y}$ and the above-mentioned prior distribution, the posterior distribution can be derived from the Bayes formula as follows:

$$
p(\boldsymbol{\theta}, \boldsymbol{\alpha}, \sigma \mid \mathbf{y})=p(\boldsymbol{\theta} \mid \mathbf{y}, \boldsymbol{\alpha}, \sigma) p(\boldsymbol{\alpha}, \sigma \mid \mathbf{y})
$$

$$
\left(\begin{array}{llll}
3 & 0 & \boldsymbol{\Sigma}
\end{array}\right)=\left(\sigma^{-2} \boldsymbol{\Phi}^{\mathrm{T}} \boldsymbol{\Phi}+\mathbf{A}\right)^{-1}
$$

where $\mathbf{A}=\operatorname{diag}\left(\alpha_{1}, \alpha_{2} \ldots \alpha_{N}\right)$.

The second term $p\left(\boldsymbol{\alpha}, \sigma^{2} \mid \mathbf{y}\right)$ is a posterior distribution of $\boldsymbol{\alpha}$ and $6^{-2}$. By maximizing the marginal likelihood function $p\left(\boldsymbol{\alpha}, \sigma^{2} \mid \mathbf{y}\right)$, the hyper-parameters $\boldsymbol{\alpha}$ and $6^{-2}$ can be estimated. Consequently, both $\boldsymbol{\alpha}$ and $6^{-2}$ obey the same distribution. The logarithm edge likelihood function $L\left(\boldsymbol{\alpha}, \sigma^{-2}\right)$ of $\boldsymbol{\alpha}$ and $\sigma^{-2}$ can be described as:

$$
\begin{array}{rl}
L\left(\boldsymbol{\alpha}, \sigma^{-2}\right) & =\log p\left(\mathbf{y} \mid \boldsymbol{\alpha}, \sigma^{-2}\right) \\
=-1 & 1 M \log 2 \pi+\log \mathbf{C} \\
& \left.+\mathbf{y}^{\mathrm{T}} \mathbf{C}^{-\mathrm{y}}\right] 2
\end{array}
$$

where $\mathbf{C}=\sigma^{2} \mathbf{E}+\boldsymbol{\Phi} \mathbf{A}^{-1} \boldsymbol{\Phi}^{\mathrm{T}}$, and $M$ represents the length

of the measurement data.

In reference [47], a fast RVM algorithm was proposed to greatly reduce the burden of computation and storage. It uses the independent correlations among elements of hyperparameter $\boldsymbol{\alpha}$ to decompose $\boldsymbol{C}$ into:

$$
\begin{aligned}
& =\oiint^{2} \quad \not \quad \alpha_{-}^{1} \phi_{\mathrm{T}_{+}} \phi_{-}^{1} d \bar{c} \\
& =\mathbf{C}+\stackrel{j}{j \neq i}_{i} \\
& { }^{1} \phi_{i i} \phi^{\mathrm{T}}
\end{aligned}
$$$$
(3
$$

where $\mathbf{C}_{-i}$ represents the matrix after removing the $i$ th basis vector $O_{i}$. Solving (32)

$$
\mathbf{C}_{-1}=\mathbf{C}_{-1-i} \text { through Woodbury's }
$$
formula [48]:

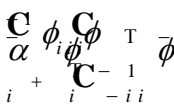

Furthermore, the determinant of $\mathbf{C}$ is:

The posterior density function consists of two parts in which the first term $p\left(\boldsymbol{\theta} \mid \mathbf{y}, \boldsymbol{\alpha}, 6^{2}\right)$ is a multi-dimensional Gaussian distribution whose mean and variance are:

$$
\boldsymbol{\mu}=\boldsymbol{\Sigma} \boldsymbol{\Phi} \mathbf{y}
$$


$\alpha_{i}^{-1} \phi_{i}^{\mathrm{T}}{ }_{i}^{-1}{ }_{-i} \phi{ }_{i}$

Substituting (34) into (31) treats $L$ as a function of $\boldsymbol{\alpha}$ : 1

2

$$
L(\boldsymbol{\alpha})=-\ulcorner M \log (2 \pi)+\log
$$

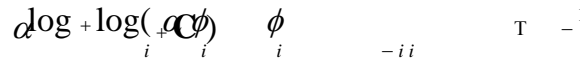

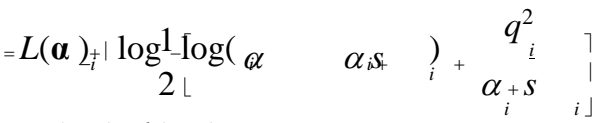

$$
\begin{aligned}
& =L(\boldsymbol{\alpha})+l(\alpha)
\end{aligned}
$$

(36)

$$
q_{i}={ }^{\mathrm{T}} \phi_{i}^{-} \mathbf{C}_{-i} \mathbf{y}
$$

where $s i$ represents the sparsity factor and $q_{i}$ represents the mass factor. Therefore, the objective function $L(\boldsymbol{\alpha})$ is translated into the sum of $L\left(\boldsymbol{\alpha}_{-i}\right)$ and $l\left(\alpha_{i}\right)$, both of which are independent.

The analysis of $l\left(\alpha_{i}\right)$ in [47] shows that $L(\boldsymbol{\alpha})$ has a unique maximum with regard to $\alpha_{i}: \alpha_{i}=s i^{2} /\left(q i^{2}-s i\right)$ when $q i^{2}>s i$, and $\alpha_{i}$ $=\infty$ when $q i^{2} \leq s i$. According to (29) and (30), $\boldsymbol{\mu}$ and $\boldsymbol{\Sigma}$ can be obtained, and the mean $\boldsymbol{\mu}$ of the posterior estimation can be regarded as estimation of the sparse vector $\boldsymbol{\theta}$. A few $\alpha_{i}$ converge to the finite value, and the corresponding $O_{i}$ is not 0 ; the remaining $\alpha_{i}$ will tend to infinity, and the corresponding $O_{i}$ is approximately 0 .

In summary, the BCS algorithm can reconstruct a sufficiently sparse signal $\boldsymbol{\theta}$, which almost matches reality. For the undetermined equations (20), the BCS algorithm can fix the

$\mathbf{C}=\mathbf{C}_{-i} 1+$

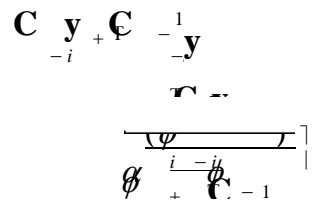

$l \quad \phi \perp \mathbf{C}^{-} \phi$ 
target 1 ; and the reduction of the false judgment can easily happen in traditional impedance estimation methods.

\section{Fault location steps}

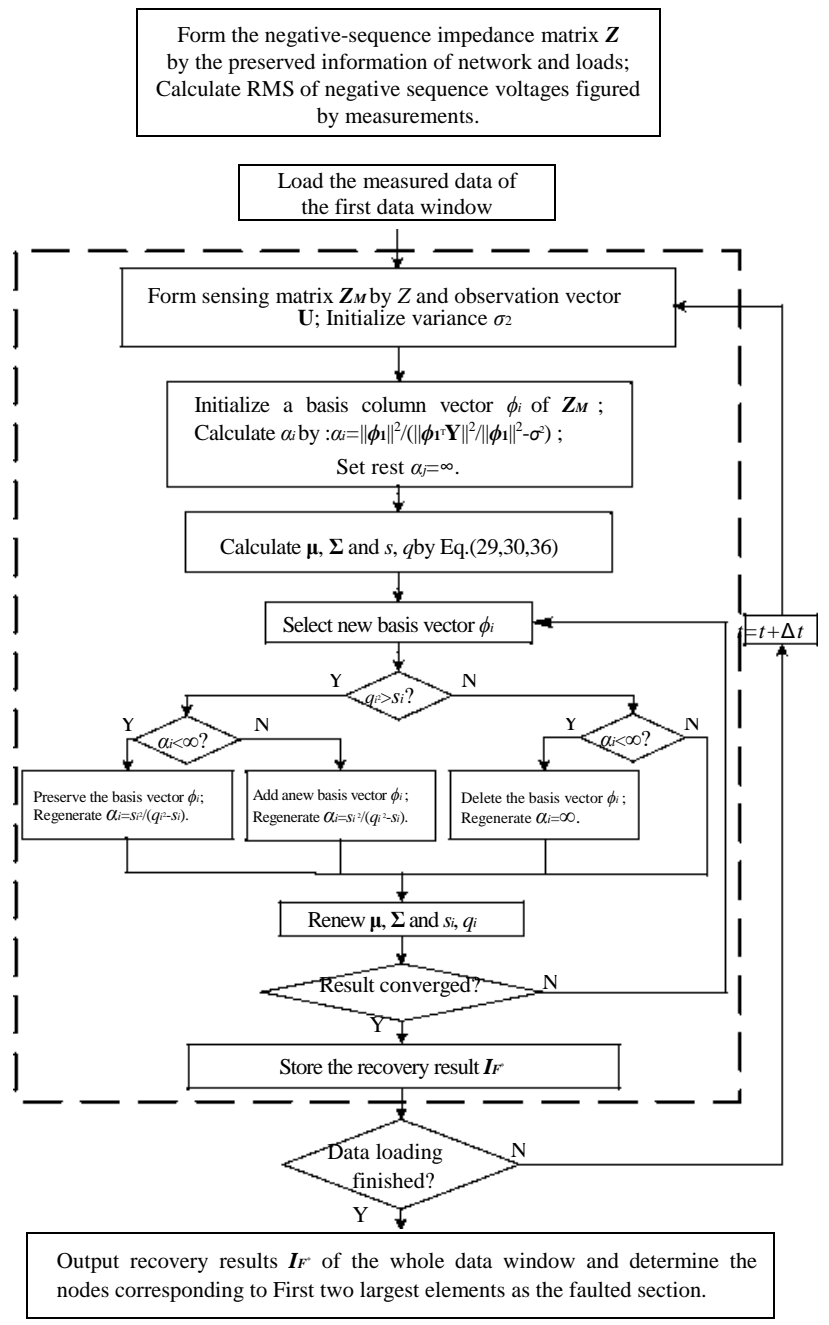

Fig. 3. Flowchart of the proposed fault-section location method.

The steps of the proposed fault-section location method are as follows.

Step 1: Formation of the negative sequence impedance matrix $\boldsymbol{Z}$ based on the network and load parameters of the distribution system. Extraction of the corresponding matrix $\boldsymbol{Z}_{\boldsymbol{M}}$ from $\boldsymbol{Z}$ according to the allocation of IEDs.

Step 2: After a fault is detected, the vector $\boldsymbol{U}_{\boldsymbol{M}}$ can be obtained based on the amplitudes of the negative sequence voltages, calculated from the measured post-fault voltages obtained from IEDs.

Step 3: Restructure of vector 1 ; by processing $\boldsymbol{Z}_{\boldsymbol{M}}$ and $\boldsymbol{U}_{\boldsymbol{M}}$ through BCS algorithm.

Step 4: Loading of the voltage amplitude data for the next sampling period and repeating steps 2-3 until all samplings within the data window have been processed.

Step 5: Comprehensively consider the location results in multiple periods to reduce misjudgment. The fault is regarded to have occurred between the two adjacent nodes whose corresponding elements in the vector 1; are the first two largest.
The flowchart of the proposed fault-section location method is shown in Fig. 3.

\section{SIMULATION AND EXPERIMENTAL TESTS}

To validate the proposed method, both simulation and experimental tests have been conducted on the US PG\&E 69nodes distribution system, as shown in Fig. 4. The network is connected to the external grid at node 1 , with a reference voltage of $12.66 \mathrm{kV}$. The detail parameters of the system, such as line impedances and loads are given in Table VII in the Appendix

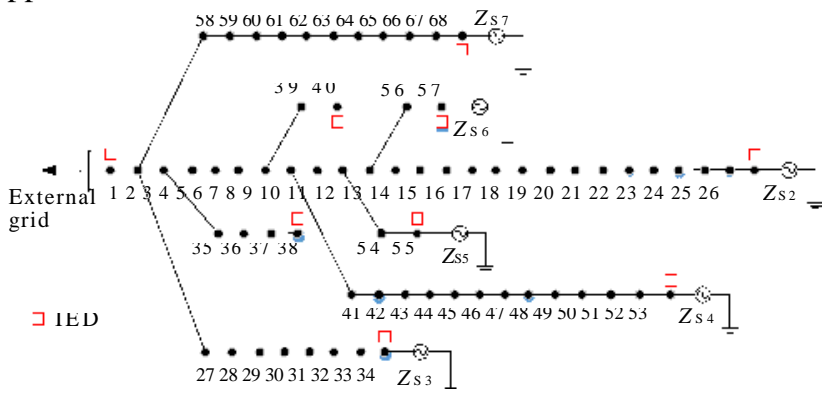

Fig. 4. US PG\&E 69-nodes distribution system diagram.

Distributed generations are added arbitrarily at nodes 26,34 , $53,55,57$, and 68 to simulate a multi-DGs condition. Based on the analysis in part B of section II, voltage measurements are obtained from IEDs installed at nodes 1, 26, 34, 38, 40, 53, 55, 57 , and 68 . The sampling frequency is $4 \mathrm{kHz}$.

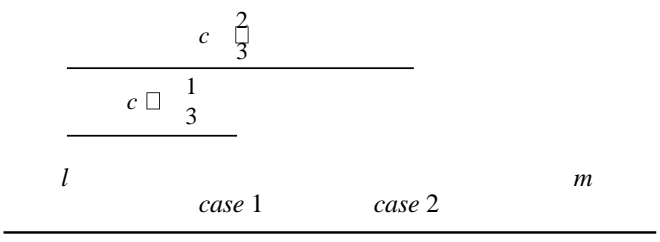

Fig. 5. Positions of fault points in different cases.

Positions of fault points are shown in Fig. 5, where $c$ represents the length percentage of distance between the fault point and node. On each line, there are set two points, with 134 points in total.

The experimental test system is shown in Fig. 6, and is composed of the main controller, PWM generator, monitor, and real time digital simulator (RTDS). The role of these components can be found in [49]. In this study, parameters of the test model are stored in various RTDS cards which constitute the RTDS processor. A DSP/FPGA combined structure is adopted in the main controller to implement the fault location algorithm based on the modular programming method. 


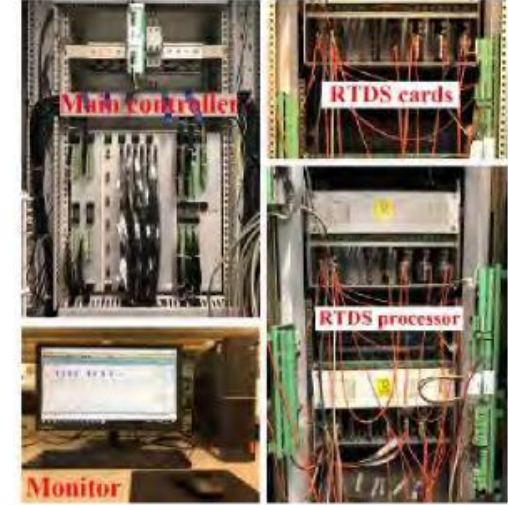

Fig. 6. Experimental test system based on RTDS.

\section{A. Influence offault type and resistance}

In this part, the proposed method is tested under various fault types and grounding resistances. Experimental explorations indicated that two types of location results exist: Type I, faulted line section is correctly identified; Type II, location is judged as the section adjacent to the faulted section. Location results are shown in Table I. Simulation results are shown in Table IV, which differs slightly from the experimental results.

$$
\text { TABLE I }
$$

\section{FAULT LOCATION RESULTS}

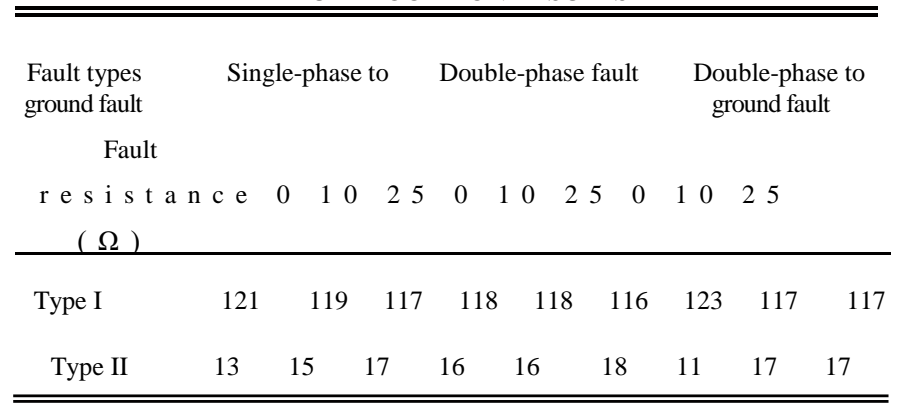

The numeric results shown in Table I indicate the amounts of different location results. The correct location percentages range from $90.30 \%$ to $93.28 \%$. The erroneous location is deemed to be affected by the parameters of lines. This implies that if the impedance of the faulted line is much smaller (about 1/30) than the adjacent line impedance, location tends to be identified incorrectly. Furthermore, the failing can be avoided by adding IEDs, according to the statistical results.

One of the location cases is that a double-phase to ground fault with fault resistance of $25 \Omega$ happened between nodes 43 and 44, and the location result is drawn in Fig. 7. The node number is plotted on the $\mathrm{X}$-axis, the time is plotted on the $\mathrm{Y}$ axis, and the elements value of $\square \square *$ is plotted on the vertical $\mathrm{Z}$-axis against the $\mathrm{XY}$ plane $(5 \mathrm{~ms}$ with a $4 \mathrm{kHz}$ sampling frequency). Fig. 7 shows that there are only two nonzero values at number 43 and 44 during the sampling time, indicating that the location result is correct.

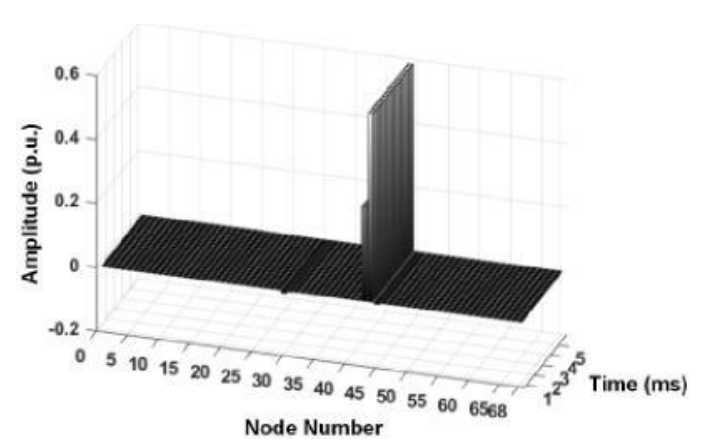

Fig. 7. Location result of the double-phase to ground fault occurred on line 4344 fault with a fault resistance of $25 \Omega$.

The vertical axis in Fig. 7 shows the computing values of virtual currents, the ordinate shows the time where the starting point refers to the starting of sampling $(0.75 \mathrm{~ms}$ after fault occurrence), and the axis of abscissae shows the nodes numbers. Figs. 8-13 have been depicted in the same way.

\section{B. Influence of noise}

Measurement accuracy is typically affected by noises generated by environmental factors [50]. In this section, the performance of the method is studied under noise. The location results of experimental tests with $30 \mathrm{~dB}$ Gaussian white noise added in measured voltages are shown in Table II. The corresponding simulation results are shown in Table $\mathrm{V}$ of the Appendix.

TABLE II

FAULT LOCATION RESULTS UNDER NOISE CONDITION

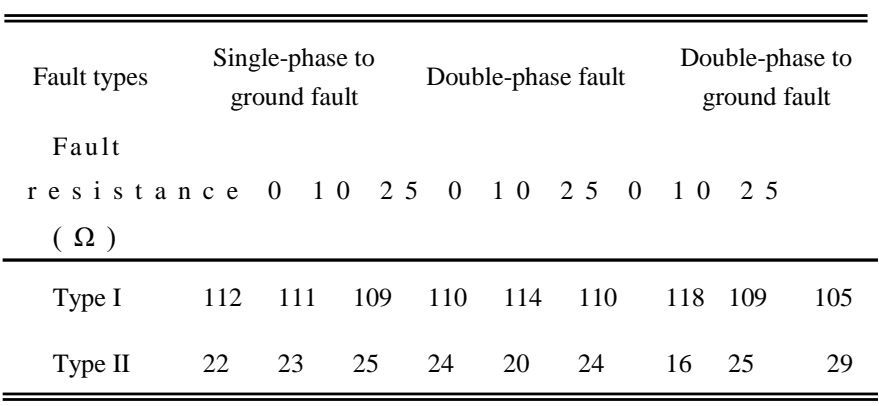

The numeric results in Table II match those presented in Table I. Table II shows that, in response to $30 \mathrm{~dB}$ noise, the proposed method still manages to obtain the fault location. At present, the error of voltage measurement devices is mostly below $0.1-0.5 \%$. Therefore, the anti-noise ability of the method can meet the requirement of practical application. The BCS algorithm provides the main contribution to decrease the effect of noise. As its sole superiority, BCS algorithm considers noise; therefore, not only the negative sequence current but also the noise can be estimated simultaneously with the iteration of the algorithm [51]. This ability equips the BCS algorithm with very strong robustness, which is also why this study chose the BCS algorithm instead of other CS algorithms. 


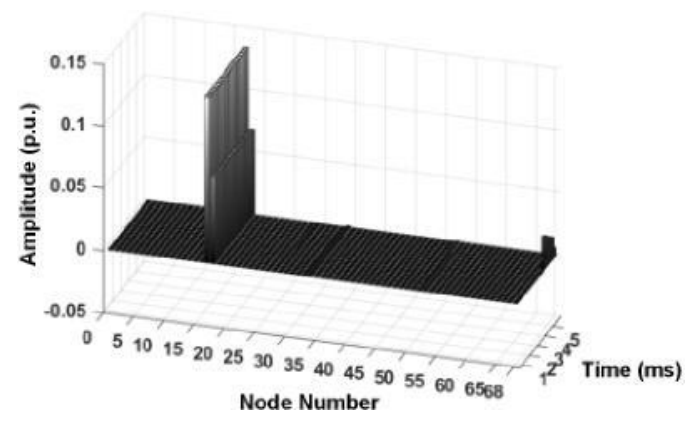

Fig. 8. Location result of single-phase to ground fault occurred on line 17-18 fault under $30 \mathrm{~dB}$ noise.

One of the location cases is that a single-phase to ground fault with a fault resistance of $10 \Omega$ occurred between nodes 17 and 18 in response to $30 \mathrm{~dB}$ noise, the recovered result $I \square *$ was drawn in Fig. 8. The fault can be determined to have occurred between nodes 17 and 18 .

\section{Influence of load and line parameter alteration}

The load and line parameters are relevant to the impedance matrix; therefore, it is necessary to measure their impact on the method. Loads may fluctuate between $80 \%$ and $120 \%$ of the original value. In this section, the proposed method was tested under the different load conditions. Experimental results of double-phase to ground under the load changed condition is shown in Table III; changes of load slightly abate the correctlocation rate. The corresponding simulation results are shown in Table VI of the Appendix.

TABLE III

FAULT LOCATION RESULTS OF DOUBLE PHSE TO GROUND FAULT WITH LOAD CHANGE

\begin{tabular}{ccccc}
\hline \hline Total load / Original load & $80 \%$ & $90 \%$ & $100 \%$ & $120 \%$ \\
\hline Type I & 105 & 115 & 123 & 117 \\
Type II & 29 & 29 & 11 & 17 \\
\hline \hline
\end{tabular}

The numeric results shown in Table III have been obtained identically to Table I. The factor of line parameters has been studied in the same way. With the parameter of each line arbitrarily adjusted from 0.9 to 1.1 times of the original value, most cases were not affected except for few cases that have been identified as Type II, which could be overwhelmed if extra IEDs were to be added near the faulted section.

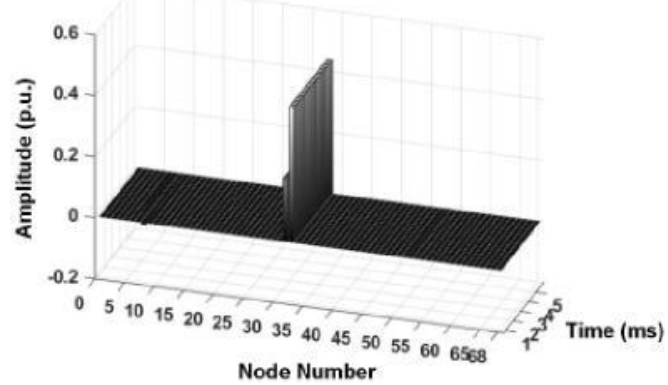

Fig. 9. Location result of double-phase fault occurred on line 32-33 fault with line parameters changing.
One of the location cases is that a double-phase fault occurred between nodes 32 and 33, at a fault resistance of $10 \Omega$, with the line parameters modified by the above procedure. The recovered result $I \square *$ is shown in Fig. 9, from which the faulted section was correctly identified.

\section{Influence of unbalanced condition}

Distribution networks are normally unbalanced among three phases. In this section, the performance of the proposed method under the unbalanced condition is discussed. It should be noted in advance, that the unbalanced condition in the high- and middle-voltage (above $10 \mathrm{kV}$ ) distribution networks, studied in this paper, is normally caused by the connected unsymmetrical loads instead of the grid itself.

To imitate the unbalanced condition, the largest loads connected at nodes $49,38,37,52,10,11,20,15,59$, and 36 have been adjusted by increasing the load of the arbitrary phase by $20 \%$. The allocation of IEDs, fault positions, and other settings remain. The results of the simulation and experimental tests validate that the proposed method performs effectively and achieves high-accuracy. The following shows the location result of a case where double-phase to ground fault occurred on the line 15-16 under the unbalanced condition.

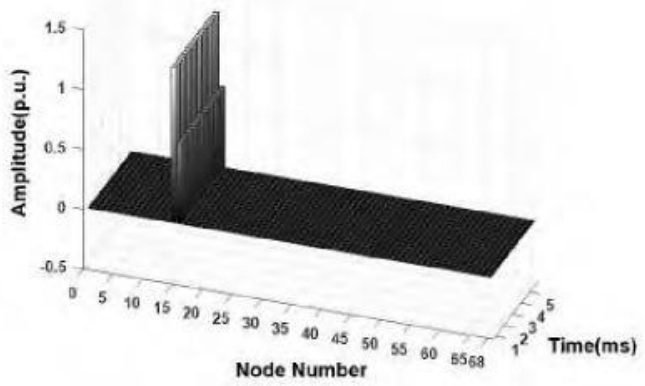

Fig. 10. Location result of a fault that occurred on line 15-16 under unbalanced-phases condition.

The tests indicate that the proposed method works normally in networks where the tri-phase unbalance percent is below $20 \%$. Additional work shows that in extremity, such as if one phase of the load is lost, the method might be affected.

\section{E. Influence of the IED allocation}

According to the analysis in part B of section II, it is essential that at least one IED is allocated respectively upstream and downstream of the faulted area to guarantee validity of the proposed method. If this allocation cannot be satisfied, the location method may have decreased correctness. In this section, the influence of IED allocation is investigated.

For the case mentioned in part A of section III, the location result is correct as shown in Fig. 4 with original IED allocation (installed at nodes $1,26,34,38,40,53,55,57$, and 68). Then, the IED is installed at node 53 to node 41 and the result is shown as Fig. 11, which is a false location. Therefore, it is recommended that IEDs must be allocated at the end of the network branches, closest to the power supplies. 


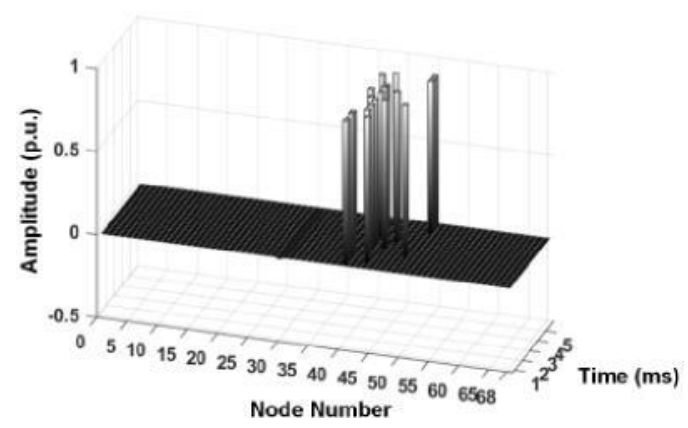

Fig. 11. Location result of double-phase to ground fault that occurred on line 43-44 with altered IEDs allocation.

In addition to the IED allocation problem, the minimum prerequisite number of IEDs also needs to be discussed. According to the theory of BCS, the minimum number of IEDs should satisfy condition (37) to guarantee the feasibility of sparse reconfiguration [52]:

$$
M \square K \ln (K)
$$

Where $K$ represents the possible number of non-zero elements of $1 \square *, N$ represents the number of nodes, and $M$ represents the number of IEDs in the network. In the PG\&E 69-nodes system, dimension of $1 \square *$ is 68 (excluding the reference node), and the number of non-zero elements is generally 2 . It can be obtained from (37) that at least seven IEDs are needed for the proposed method. However, there are nine distributaries in the system; therefore, at least nine IEDs are needed in total. In summary, the IED allocation installed at nodes 1, 26, 34, 38, 40, $53,55,57$, and 68 can fulfil the location requirement.

\section{F. Influence of harmonics}

In theory, the proposed method uses the negative-sequence impedance matrix and voltages to estimate the fault location which are derived from the fundamental frequency component. Therefore, harmonics will likely not affect fault location. To verify this assertion, a harmonics condition has been emulated in the modified PG\&E 69-nodes distribution system in PSCAD/EMTDC. One harmonic source is connected at node 49 and the current magnitude of the 5 th harmonic order $(250 \mathrm{~Hz})$ is set as $1 \mathrm{~A}$.

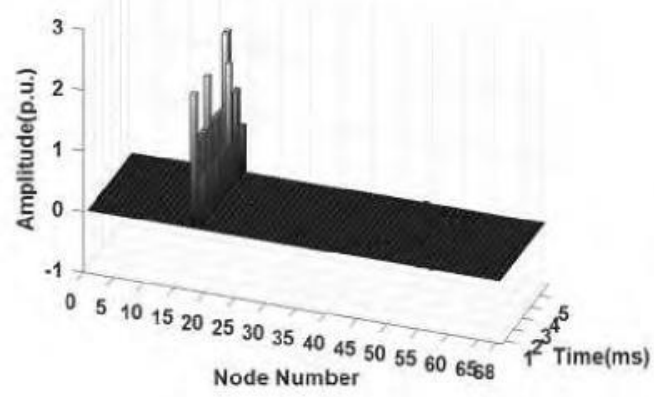

Fig. 12. Location result of double-phase to ground fault that occurred on line 18-19 under harmonics condition.
Fig. 12 shows that the simulation results indicate that the harmonics do not affect the proposed method. The location results of other cases retain the condition as depicted in Table I.

\section{G. Influence ofphotovoltaic $(P V)$ power plant}

In this section, the effect of inverter interfaced DGs is studied. For convenience, only a PV power plant is involved in this thesis.

The control strategy of PV is generally to suppress the negative-sequence injected current for smooth FRT [53]. Therefore, it seems to be that access of PV would not make any difference to the proposed method. In fact, negative-sequence currents are outputted from PV inverters. However, the currents are rather small compared to the fault negative current; therefore, this suggests that PV will not impact the location method. To verify this, three $1 \mathrm{MW}-\mathrm{PV}$ power stations are connected to nodes 29,31 , and 45 , respectively.

For the case that a double-phase fault with a fault resistance of $25 \Omega$ occurred between nodes 32 and 33, $1 \square *$ is as shown in Fig. 13. Although the access of the PV dose influences the reconstruction result, the faulted section can still be correctly identified.

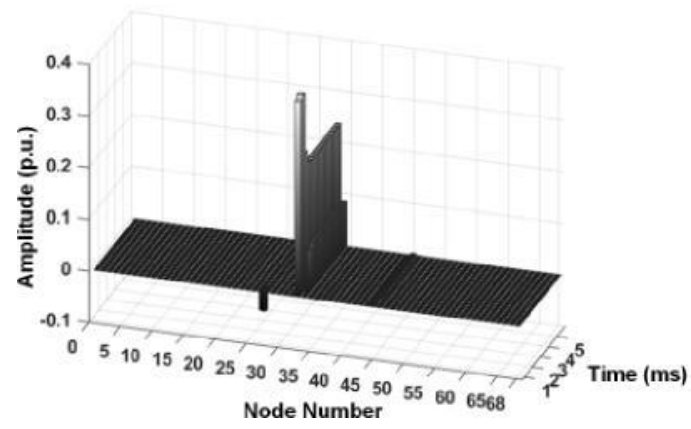

Fig. 13. Location result of double-phase to ground fault that occurred on line 32-33 with PV access.

\section{H. Discussion of threshold of recovered virtual currents}

Additional discussion upon the threshold of the recovered virtual currents, i.e. the amplitudes in Fig. $7-13$ is presented in this section.

With regard to the recovered result $1 \square *$, two nonzero items of the vector should be relative to both nodes of the identified faulted line section. Supposing that there are more items in the nonzero vector, it is significant to decide which two should be taken as nodes corresponding to the faulted section. To distinguish the two items from others, the differential degree between these and the others is used as threshold in this study. The differential degree is defined as ratio of the lower value of the biggest items and the highest value of others. For a specific case, if the differential degree is obtained above a certain threshold, the location result can be identified from $1 \square *$.

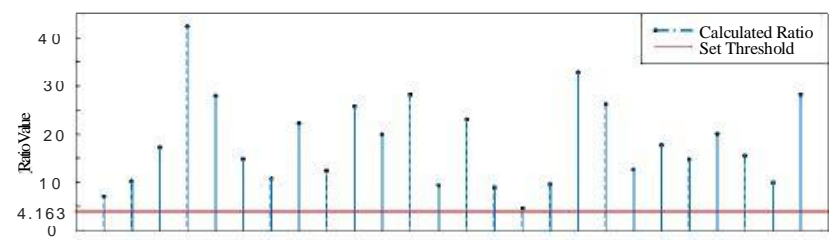

Fig. 14. Calculated ratio of currents and set threshold. 
In Fig. 14, the ordinate shows ratios of the lower value of the biggest items and the highest value of others, and the abscissae shows the different conditions. As shown in the statistical chart, the minimum differential degree is 4.626 . In consideration of error, the threshold can be defined as 4.163 , which equals the minimum ratio multiplied by a coefficient 0.9 to distinguish all valid results from the invalid. Therefore, the set threshold indicates that only if the differential degree of values in a location result is above 4.163 the result could be seen as discernible and valid.

\section{CONCLUSION}

In this study, a sparse measurements-based fault-section location method aimed at asymmetrical faults is proposed. This method is applicable for distribution networks with DGs. Utilizing the amplitudes simplifies the algorithm, and the feasibility of the simplification has been demonstrated. To recover $\square \square *$ through sparse measurements, the theory of BCS was introduced to solve the underdetermined function. The traits of the proposed method verified by experimental tests and simulation results can be summarized as follows:

- The proposed method provides robust location results under a variety of asymmetrical fault scenarios.

- Big fault resistance (100 Q) and high noisy (30 dB) do not invalidate the method.

- Subacute changes of line and load parameters do not invalidate the method.

- Few IEDs allocated in the terminal buses are needed for the fault location; therefore, the implementation cost is low. The meter location was optimized in the methodology, which is verified in the simulation.

- This also proved that the proposed method is applicable with the introduction of inverter interfaced DGs, which shows the engineering value of this method.

\section{APPENDIX}

Fault location results of simulation verification under various conditions are shown below. Please note that each tabulation is corresponding to the result of experimental tests, i.e., Table IV shows the simulation corresponding to the various fault types and resistances shown in Table I, Table V and VI are respectively corresponding to Tables II and III.

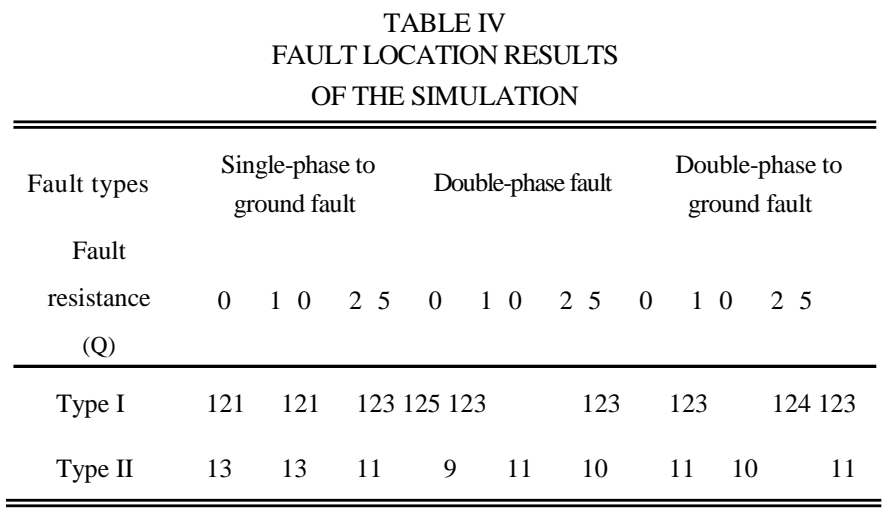

TABLE V

FAULT LOCATION RESULTS UNDER NOISE CONDITION OF SIMULATION

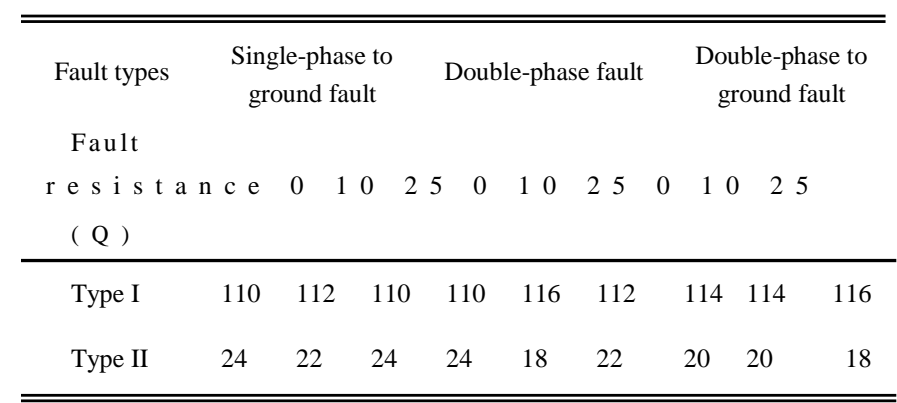

TABLE VI

FAULT LOCATION RESULTS OF DOUBLE PHSE TO GROUND FAULT WITH LOAD CHANGE IN THE SIMULATION

\begin{tabular}{ccccc}
\hline \hline Total load/ Original load & $80 \%$ & $90 \%$ & $100 \%$ & $120 \%$ \\
\hline Type I & 118 & 117 & 123 & 118 \\
Type II & 16 & 17 & 11 & 16 \\
\hline \hline
\end{tabular}

The distribution system used in this study is the US PG\&E 69-nodes. The detail parameters of lines and loads are shown in Table VII. The impedances are per-unit values, the voltage reference is $12.66 \mathrm{kV}$, and the power reference is $10 \mathrm{MVA}$. The column "Load on $j$ " indicates the complex power of the load connected at end bus $j$. Among the lines, 69-73 are loop switch branches. The gross overall load of the system is $3802.19+$ j2694.60 kVA.

TABLE VII

PARAMETERS OF THE FORMULATION SYSTEM

\begin{tabular}{|c|c|c|c|c|c|c|c|c|c|}
\hline Line & $\begin{array}{l}\text { Head } \\
\text { bus } i\end{array}$ & $\begin{array}{l}\text { End } \\
\text { bus } j\end{array}$ & $\begin{array}{l}\text { Impedance of the line } \\
\text { (p.u.) }\end{array}$ & $\begin{array}{c}\text { Load at } j \\
(\mathrm{kVA})\end{array}$ & Line & $\begin{array}{l}\text { Head } \\
\text { bus } i\end{array}$ & $\begin{array}{l}\text { End } \\
\text { bus } j\end{array}$ & $\begin{array}{l}\text { Impedance of the line } \\
\text { (p.u.) }\end{array}$ & $\begin{array}{c}\text { Load at } j \\
(\mathrm{kVA})\end{array}$ \\
\hline 1 & 1 & 2 & $0.0050+\mathrm{j} 0.0012$ & 0 & 38 & 61 & 62 & $0.0304+\mathrm{j} 0.0355$ & $24+\mathrm{j} 17$ \\
\hline 2 & 2 & 3 & $0.0050+\mathrm{j} 0.0012$ & 0 & 39 & 62 & 63 & $0.0018+\mathrm{j} 0.0021$ & $24+\mathrm{j} 17$ \\
\hline 3 & 3 & 4 & $0.0015+\mathrm{j} 0.0036$ & 0 & 40 & 63 & 64 & $0.7283+\mathrm{j} 0.8509$ & $1.2+\mathrm{j} 1$ \\
\hline 4 & 4 & 5 & $0.0251+\mathrm{j} 0.0294$ & 0 & 41 & 64 & 65 & $0.3100+\mathrm{j} 0.3623$ & 0 \\
\hline 5 & 5 & 6 & $0.3660+\mathrm{j} 0.1864$ & $2.6+j 2.2$ & 42 & 65 & 66 & $0.0410+\mathrm{j} 0.0478$ & $6+j 4.3$ \\
\hline 6 & 6 & 7 & $0.3811+\mathrm{j} 0.1941$ & $40.4+\mathrm{j} 30$ & 43 & 66 & 67 & $0.0092+\mathrm{j} 0.0116$ & 0 \\
\hline 7 & 7 & 8 & $0.0922+\mathrm{j} 0.0470$ & $75+\mathrm{j} 54$ & 44 & 67 & 68 & $0.1089+\mathrm{j} 0.1373$ & $39.22+\mathrm{j} 26.3$ \\
\hline 8 & 8 & 9 & $0.0493+\mathrm{j} 0.0251$ & $30+\mathrm{j} 22$ & 45 & 68 & 69 & $0.0009+\mathrm{j} 0.0012$ & $39.22+\mathrm{j} 26.3$ \\
\hline 9 & 9 & 10 & $0.8190+\mathrm{j} 0.2707$ & $28+\mathrm{j} 19$ & 46 & 4 & 36 & $0.0034+\mathrm{j} 0.0084$ & 0 \\
\hline 10 & 10 & 11 & $0.1872+\mathrm{j} 0.0691$ & $145+\mathrm{j} 104$ & 47 & 36 & 37 & $0.0851+\mathrm{j} 0.2083$ & $79+\mathrm{j} 56.4$ \\
\hline
\end{tabular}




\begin{tabular}{|c|c|c|c|c|c|c|c|c|c|}
\hline 11 & 11 & 12 & $0.7114+\mathrm{j} 0.2351$ & $145+\mathrm{i} 104$ & $\overline{448}$ & 37 & 38 & $0.2898+\mathrm{j} 0.7091$ & $384.70+\mathrm{i} 274.5$ \\
\hline 12 & 12 & 13 & $1.0300+\mathrm{j} 0.3400$ & $8+\mathrm{j} 5.5$ & 49 & 38 & 39 & $0.0822+\mathrm{j} 0.2011$ & $384.70+\mathrm{j} 274.5$ \\
\hline 13 & 13 & 14 & $1.0440+\mathrm{j} 0.3450$ & $8+\mathrm{j} 5.5$ & 50 & 8 & 40 & $0.0928+\mathrm{j} 0.0473$ & $40.5+\mathrm{j} 28.3$ \\
\hline 14 & 14 & 15 & $1.0580+\mathrm{j} 0.3496$ & 0 & 51 & 40 & 41 & $0.3319+\mathrm{j} 0.1114$ & $3.6+\mathrm{j} 2.7$ \\
\hline 15 & 15 & 16 & $0.1966+\mathrm{j} 0.0650$ & $45.5+\mathrm{j} 30$ & 52 & 9 & 42 & $0.1740+\mathrm{j} 0.0886$ & $4.35+\mathrm{j} 3.5$ \\
\hline 16 & 16 & 17 & $0.3744+\mathrm{j} 0.1238$ & $60+j 35$ & 53 & 42 & 43 & $0.2030+\mathrm{j} 0.1034$ & $26.4+j 19$ \\
\hline 17 & 17 & 18 & $0.0047+\mathrm{j} 0.0016$ & $60+j 35$ & 54 & 43 & 44 & $0.2842+\mathrm{j} 0.1447$ & $24+\mathrm{j} 17.2$ \\
\hline 18 & 18 & 19 & $0.3276+\mathrm{j} 0.1083$ & 0 & 55 & 44 & 45 & $0.2813+\mathrm{j} 0.1433$ & 0 \\
\hline 19 & 19 & 20 & $0.2106+j 0.0696$ & $1+\mathrm{j} 0.6$ & 56 & 45 & 46 & $1.5900+\mathrm{j} 0.5337$ & 0 \\
\hline 20 & 20 & 21 & $0.3416+j 0.1129$ & $114+\mathrm{j} 81$ & 57 & 46 & 47 & $0.7837+\mathrm{j} 0.2630$ & 0 \\
\hline 21 & 21 & 22 & $0.0140+\mathrm{j} 0.0046$ & $5.3+\mathrm{j} 3.5$ & 58 & 47 & 48 & $0.3042+\mathrm{j} 0.1006$ & $100+\mathrm{j} 72$ \\
\hline 22 & 22 & 23 & $0.1591+\mathrm{j} 0.0526$ & 0 & 59 & 48 & 49 & $0.3861+\mathrm{j} 0.1172$ & 0 \\
\hline 23 & 23 & 24 & $0.3463+\mathrm{j} 0.1145$ & $28+\mathrm{j} 20$ & 60 & 49 & 50 & $0.5075+\mathrm{j} 0.2585$ & $1244+\mathrm{j} 888$ \\
\hline 24 & 24 & 25 & $0.7488+\mathrm{j} 0.2457$ & 0 & 61 & 50 & 51 & $0.0974+\mathrm{j} 0.0496$ & $32+j 23$ \\
\hline 25 & 25 & 26 & $0.3089+\mathrm{j} 0.1021$ & $14+\mathrm{j} 10$ & 62 & 51 & 52 & $0.1450+\mathrm{j} 0.0738$ & 0 \\
\hline 26 & 26 & 27 & $0.1732+\mathrm{j} 0.0572$ & $14+\mathrm{j} 10$ & 63 & 52 & 53 & $0.7105+\mathrm{j} 0.3619$ & $227+\mathrm{j} 162$ \\
\hline 27 & 3 & 28 & $0.0044+\mathrm{j} 0.0108$ & $26+j 18.6$ & 64 & 53 & 54 & $1.041+\mathrm{j} 0.5302$ & $59+\mathrm{j} 42$ \\
\hline 28 & 28 & 29 & $0.0640+\mathrm{j} 0.1565$ & $26+j 18.6$ & 65 & 11 & 55 & $0.2012+\mathrm{j} 0.0611$ & $18+\mathrm{j} 13$ \\
\hline 29 & 29 & 30 & $0.3978+\mathrm{j} 0.1315$ & 0 & 66 & 55 & 56 & $0.0047+\mathrm{j} 0.0014$ & $18+\mathrm{j} 13$ \\
\hline 30 & 30 & 31 & $0.0702+\mathrm{j} 0.0232$ & 0 & 67 & 12 & 57 & $0.7394+\mathrm{j} 0.2444$ & $28+\mathrm{j} 20$ \\
\hline 31 & 31 & 32 & $0.3510+\mathrm{j} 0.1160$ & 0 & 68 & 57 & 58 & $0.0047+\mathrm{j} 0.0016$ & $28+\mathrm{j} 20$ \\
\hline 32 & 32 & 33 & $0.8390+\mathrm{j} 0.2816$ & $14+\mathrm{j} 10$ & 69 & 11 & 66 & $0.5000+\mathrm{j} 0.5000$ & \\
\hline 33 & 33 & 34 & $1.7080+\mathrm{j} 0.5646$ & $19.5+\mathrm{j} 14$ & 70 & 13 & 20 & $0.5000+\mathrm{j} 0.5000$ & \\
\hline 34 & 34 & 35 & $1.4740+\mathrm{j} 0.4873$ & $6+j 4$ & 71 & 15 & 69 & $1.0000+\mathrm{j} 1.0000$ & \\
\hline 35 & 3 & 59 & $0.0044+\mathrm{j} 0.0108$ & $26+j 18.55$ & 72 & 27 & 54 & $1.0000+\mathrm{j} 1.0000$ & \\
\hline 36 & 59 & 60 & $0.0640+\mathrm{j} 0.1565$ & $26+\mathrm{j} 18.55$ & 73 & 39 & 48 & $2.0000+\mathrm{j} 2.0000$ & \\
\hline 37 & 60 & 61 & $0.1053+\mathrm{j} 0.1230$ & 0 & & & & & \\
\hline
\end{tabular}

\section{REFERENCES}

[1] M. Kezunovic, "Smart Fault Location for Smart Grids," in IEEE Transactions on Smart Grid, vol. 2, no. 1, pp. 11-22, March 2011

[2] T. Takagi, Y. Yamakoshi, J. Baba, K. Uemura and T. Sakaguchi, "A New Alogorithm of an Accurate Fault Location for EHV/UHV Transmission Lines: Part I - Fourier Transformation Method," in IEEE Transactions on Power Apparatus and Systems, vol. PAS-100, no. 3, pp. 1316-1323, March 1981.

[3] M. S. Choi, S. J. Lee, D. S. Lee and B. G. Jin, "A new fault location algorithm using direct circuit analysis for distribution systems," in IEEE Transactions on Power Delivery, vol. 19, no. 1, pp. 35-41, January 2004

[4] G. Morales-Espana, J. Mora-Florez and H. Vargas-Torres, "Elimination of Multiple Estimation for Fault Location in Radial Power Systems by Using Fundamental Single-End Measurements," in IEEE Transactions on Power Delivery, vol. 24, no. 3, pp. 1382-1389, July 2009.

[5] G. Manassero, S. G. Di Santo and L. Souto, "Heuristic Method for Fault Location in Distribution Feeders With the Presence of Distributed Generation," in IEEE Transactions on Smart Grid, vol. 8, no. 6, pp. 28492858, November 2017.

[6] K. Jia, T. Bi, Z. Ren, D. W. P. Thomas and M. Sumner, "High Frequency Impedance Based Fault Location in Distribution System With DGs," in IEEE Transactions on Smart Grid, vol. 9, no. 2, pp. 807-816, March 2018.

[7] C. Orozco-Henao, A. S. Bretas, R. Chouhy-Leborgne, A. R. HerreraOrozco and J. Marín-Quintero, "Active distribution network fault location methodology: a minimum fault reactance and Fibonacci search approach," in International Journal ofElectrical Power \& Energy Systems, vol. 84, pp. 232-241, January 2017.

[8] K. Jia, T. Bi, B. Liu, E. Christopher, D. W. P. Thomas and M. Sumner, "Marine Power Distribution System Fault Location Using a Portable Injection Unit," in IEEE Transactions on Power Delivery, vol. 30, no. 2, pp. 818-826, April 2015.

[9] J. Duan, K. Zhang and L. Cheng, "A Novel Method of Fault Location for Single-Phase Microgrids," in IEEE Transactions on Smart Grid, vol. 7, no. 2, pp. 915-925, March 2016.

[10] S. Masuda, H. Nakatani and S. Hosokawa, "Modification in Waveform of Traveling Pulse Voltages Due to Corona Production Along Transmission
Line," in IEEE Transactions on Industry Applications, vol. IA-21, no. 3, pp. 724-732, May 1985.

[11] J. Zhou, B. Ayhan, C. Kwan, S. Liang and W. Lee, "High-Performance Arcing-Fault Location in Distribution Networks," in IEEE Transactions on Industry Applications, vol. 48, no. 3, pp. 1107-1114, May-June 2012.

[12] R. Razzaghi, G. Lugrin, H. Manesh, C. Romero, M. Paolone and F. Rachidi, "An Efficient Method Based on the Electromagnetic Time Reversal to Locate Faults in Power Networks," in IEEE Transactions on Power Delivery, vol. 28, no. 3, pp. 1663-1673, July 2013.

[13] L. Abboud, A. Cozza and L. Pichon, "A Matched-Pulse Approach for SoftFault Detection in Complex Wire Networks," in IEEE Transactions on Instrumentation and Measurement, vol. 61, no. 6, pp. 1719-1732, June 2012.

[14] A. A. Girgis, C. M. Fallon and D. L. Lubkeman, "A fault location technique for rural distribution feeders," in IEEE Transactions on Industry Applications, vol. 29, no. 6, pp. 1170-1175, NovemberDecember 1993.

[15] T. Tayjasanant, Chun $\mathrm{Li}$ and W. Xu, "A resistance sign-based method for voltage sag source detection," in IEEE Transactions on Power Delivery, vol. 20, no. 4, pp. 2544-2551, October 2005.

[16] R. A. F. Pereira, L. G. W. da Silva, M. Kezunovic and J. R. S. Mantovani, "Improved Fault Location on Distribution Feeders Based on Matching During-Fault Voltage Sags," in IEEE Transactions on Power Delivery, vol. 24, no. 2, pp. 852-862, April 2009.

[17] P. Chen, V. Malbasa, Y. Dong and M. Kezunovic, "Sensitivity Analysis of Voltage Sag Based Fault Location With Distributed Generation," in IEEE Transactions on Smart Grid, vol.6, no. 4, pp.2098-2106, July2015.

[18] S. Lotfifard, M. Kezunovic and M. J. Mousavi, "Voltage Sag Data Utilization for Distribution Fault Location," in IEEE Transactions on Power Delivery, vol. 26, no. 2, pp. 1239-1246, April 2011.

[19] F. C. L. Trindade and W. Freitas, "Low Voltage Zones to Support Fault Location in Distribution Systems With Smart Meters," in IEEE Transactions on Smart Grid, vol. 8, no. 6, pp. 2765-2774, November 2017.

[20] J. Ge et al., "A fault location method of error correction based on ant colony algorithm for distribution network," 2017 IEEE 2nd Advanced Information Technology, Electronic and Automation Control Conference (IAEAC), Chongqing, 2017, pp. 18-21. 
[21] J. Teng, W. Huang and S. Luan, "Automatic and Fast Faulted LineSection Location Method for Distribution Systems Based on Fault Indicators," in IEEE Transactions on Power Systems, vol. 29, no. 4, pp. 1653-1662, July 2014.

[22] K. Sun, Q. Chen and Z. Gao, "An Automatic Faulted Line Section Location Method for Electric Power Distribution Systems Based on Multisource Information," in IEEE Transactions on Power Delivery, vol. 31, no. 4, pp. 1542-1551, August 2016.

[23] IEC Standard for Communication Network and Systems in Substations, IEC 61850, 1st ed., April 2003.

[24] P. Parikh, I. Voloh and M. Mahony, "Fault location, isolation, and service restoration (FLISR) technique using IEC 61850 GOOSE," 2013 IEEE Power \& Energy Society General Meeting, Vancouver, BC, 2013, pp. 16 .

[25] W. Ling, D. Liu, Y. Lu, P. Du and F. Pan, "IEC 61850 Model Expansion Toward Distributed Fault Localization, Isolation, and Supply Restoration," in IEEE Transactions on Power Delivery, vol. 29, no. 3, pp. 977-984, June 2014.

[26] S. Zubic, A. Burek, M. Krakowski, P. Balcerek and M. Fulczyk, "Utilisation of IEC61850 GOOSE for improvement of EFP sensitivity in compensated MV networks," in The Journal of Engineering, vol. 2018, no. 15, pp. 1190-1194, October 2018.

[27] M. Majidi, M. Etezadi-Amoli and M. S. Fadali, "A Sparse-Data-Driven Approach for Fault Location in Transmission Networks," in IEEE Transactions on Smart Grid, vol. 8, no. 2, pp. 548-556, March 2017.

[28] N. E. Halabi, M. García-Gracia, J. Borroy and J. L. Villa, "Current phase comparison pilot scheme for distributed generation networks protection," in Applied Energy, vol. 88, no. 12, pp. 4563-4569, December 2011.

[29] S. Mirsaeidi, D. M. Said, M. W. Mustafa, M. H. Habibuddin and K. Ghaffari, "Fault location and isolation in micro-grids using a digital central protection unit," in Renewable and Sustainable Energy Reviews, vol. 56, pp. 1-17, April 2016.

[30] A. S. Dobakhshari and A. M. Ranjbar, "A Novel Method for Fault Location of Transmission Lines by Wide-Area Voltage Measurements Considering Measurement Errors," in IEEE Transactions on Smart Grid, vol. 6, no. 2, pp. 874-884, March 2015.

[31] I. Rozenberg, Y. Beck, Y. C. Eldar and Y. Levron, "Sparse Estimation of Faults by Compressed Sensing With Structural Constraints," in IEEE Transactions on Power Systems, vol. 33, no. 6, pp. 5935-5944, November 2018

[32] M. J. B. Reddy, D. V. Rajesh, P. Gopakumar and D. K. Mohanta, "Smart Fault Location for Smart Grid Operation Using RTUs and Computational Intelligence Techniques," in IEEE Systems Journal, vol. 8, no. 4, pp. 12601271, December 2014

[33] M. M. Tawfik and M. M. Morcos, "On the use of Prony method to locate faults in loop systems by utilizing modal parameters of fault current," in IEEE Transactions on Power Delivery, vol. 20, no. 1, pp. 532-534, January 2005.

[34] M. J. B. Reddy and D. K. Mohanta, "Performance Evaluation of an Adaptive-Network-Based Fuzzy Inference System Approach for Location of Faults on Transmission Lines Using Monte Carlo Simulation," in IEEE Transactions on Fuzzy Systems, vol. 16, no. 4, pp. 909-919, August 2008.

[35] O. Babayomi, P. Oluseyi, G. Keku and N. A. Ofodile, "Neuro-fuzzy based fault detection identification and location in a distribution network," 2017 IEEE PES PowerAfrica, Accra, 2017, pp. 164-168.

[36] S. Jamali and A. Bahmanyar, "A new fault location method for distribution networks using sparse measurements," in International Journal of Electrical Power \& Energy Systems, vol. 81, pp. 459-468, October 2016.

[37] S. J. Lee et al., "An intelligent and efficient fault location and diagnosis scheme for radial distribution systems," in IEEE Transactions on Power Delivery, vol. 19, no. 2, pp. 524-532, April 2004.

[38] S. Jamali, A. Bahmanyar, and E. Bompard, "Fault location method for distribution networks using smart meters," in Measurement, vol. 102, pp. 150-157, May 2017.

[39] A. Tian, W. Li and Z. K. R. Liu, "A fault feature matching-based fault location for distribution network with DGs," 2016 IEEE Industrial Electronics and Applications Conference (IEACon), Kota Kinabalu, 2016, pp. 167-172.

[40] S. Zhou, X. R. Wang and Q. Q. Qian, "Bayesian networks based distributed fault diagnosis approach for power grids," in Power System Technology, vol. 34, no. 9, pp. 76-81. September 2011.

[41] F. C. L. Trindade, W. Freitas and J. C. M. Vieira, "Fault Location in Distribution Systems Based on Smart Feeder Meters," in IEEE
Transactions on Power Delivery, vol. 29, no. 1, pp. 251-260, February 2014.

[42]Z. Galijasevic and A. Abur, "Fault location using voltage measurements," in IEEE Transactions on Power Delivery, vol. 17, no. 2, pp. 441-445, April 2002.

[43] J. J. Grainger and W. D. Stevenson, Jr., "The Impedance Model and Network Calculations" in Power System Analysis, 17th ed, McGrawHill, Singapore, 2003, ch. 8, sec. 8.1-8.2, pp. 284-294.

[44]H. E. Brown and S. A. Sebo, "Solution of Large Networks by Matrix Methods," in IEEE Transactions on Systems, Man, and Cybernetics, vol. SMC-6, no. 5, pp. 390-391, May 1976.

[45]D. L. Donoho, "Compressed sensing," in IEEE Transactions on Information Theory, vol. 52, no. 4, pp. 1289-1306, April 2006.

[46]M. E. Tipping, "Sparse Bayesian learning and the relevance vector machine," in Journal of Machine Learning Research, vol. 1, no. 3, pp. 221-244, June 2001.

[47] A. C. Faul and M. E Tipping, "Analysis of Sparse Bayesian Learning," in Advances in Neural Information Processing Systems, vol. 14, pp. 383-389, April 2002.

[48] Golub G. H., Loan V, Matrix Computations. Maryland: Baltimore, 1996, pp. 392-396.

[49] S. Liu, T. Bi, K. Jia and Q. Yang, "Coordinated fault-ride-through strategy for doubly-fed induction generators with enhanced reactive and active power support," in IET Renewable Power Generation, vol. 10, no. 2, pp. 203-211, February 2016.

[50] M. Etezadi-Amoli, M. Ghofrani and A. Arabali, "Performance of advanced meters: Effects of different temperatures and loading conditions on meter accuracy," 2014 IEEE PES T\&D Conference and Exposition, Chicago, IL, 2014, pp. 1-5.

[51]S. D. Babacan, R. Molina and A. K. Katsaggelos, "Bayesian Compressive Sensing Using Laplace Priors," in IEEE Transactions on Image Processing, vol. 19, no. 1, pp. 53-63, January 2010.

[52] D. Mackenzie. "Compressed Sensing Makes Every Pixel Count," in What's Happening in the Mathematical Science. Providence, RI, USA: American Mathematical Society, vol. 7, 2009, pp. 114-127.

[53] J. Miret, M. Castilla, A. Camacho, L. G. d. Vicuña and J. Matas, "Control Scheme for Photovoltaic Three-Phase Inverters to Minimize Peak Currents During Unbalanced Grid-Voltage Sags," in IEEE Trans. Power Electronics, vol. 27, no. 10, pp. 4262-4271, October 2012 